\title{
Pre-clinical and clinical studies on the role of RBM3 in muscle-invasive bladder cancer: longitudinal expression, transcriptome-level effects and modulation of chemosensitivity
}

Sara Wahlin ${ }^{1 *}$, Karolina Boman ${ }^{1}$, Bruce Moran², Björn Nodin ${ }^{1}$, William M. Gallagher ${ }^{2}$, Emelie Karnevi ${ }^{1}$ and Karin Jirström ${ }^{1}$

\begin{abstract}
Background: The response to neoadjuvant cisplatin-based chemotherapy (NAC) in muscle-invasive bladder cancer (MIBC) is impaired in up to $50 \%$ of patients due to chemoresistance, with no predictive biomarkers in clinical use. The proto-oncogene RNA-binding motif protein 3 (RBM3) has emerged as a putative modulator of chemotherapy response in several solid tumours but has a hitherto unrecognized role in MIBC.

Methods: RBM3 protein expression level in tumour cells was assessed via immunohistochemistry in paired transurethral resection of the bladder (TURB) specimens, cystectomy specimens and lymph node metastases from a consecutive cohort of 145 patients, 65 of whom were treated with NAC. Kaplan-Meier and Cox regression analyses were applied to estimate the impact of RBM3 expression on time to recurrence (TTR), cancer-specific survival (CSS), and overall survival (OS) in strata according to NAC treatment. The effect of siRNA-mediated silencing of RBM3 on chemosensitivity was examined in RT4 and T24 human bladder carcinoma cells in vitro. Cellular functions of RBM3 were assessed using RNA-sequencing and gene ontology analysis, followed by investigation of cell cycle distribution using flow cytometry.

Results: RBM3 protein expression was significantly higher in TURB compared to cystectomy specimens but showed consistency between primary tumours and lymph node metastases. Patients with high-tumour specific RBM3 expression treated with NAC had a significantly reduced risk of recurrence and a prolonged CSS and OS compared to NAC-untreated patients. In high-grade T24 carcinoma cells, which expressed higher RBM3 mRNA levels compared to RT4 cells, RBM3 silencing conferred a decreased sensitivity to cisplatin and gemcitabine. Transcriptomic analysis revealed potential involvement of RBM3 in facilitating cell cycle progression, in particular $G_{1} / S$-phase transition, and initiation of DNA replication. Furthermore, siRBM3-transfected T24 cells displayed an accumulation of cells residing in the $\mathrm{G}_{1}$-phase as well as altered levels of recognised regulators of $\mathrm{G}_{1}$-phase progression, including Cyclin D1/CDK4 and CDK2.
\end{abstract}

\footnotetext{
*Correspondence: Sara.wahlin@med.lu.se

${ }^{1}$ Division of Oncology and Therapeutic Pathology, Department of Clinical

Sciences, Lund University, Lund, Sweden

Full list of author information is available at the end of the article
} original author(s) and the source, provide a link to the Creative Commons licence, and indicate if changes were made. The images or other third party material in this article are included in the article's Creative Commons licence, unless indicated otherwise in a credit line to the material. If material is not included in the article's Creative Commons licence and your intended use is not permitted by statutory regulation or exceeds the permitted use, you will need to obtain permission directly from the copyright holder. To view a copy of this licence, visit http://creativecommons.org/licenses/by/4.0/. The Creative Commons Public Domain Dedication waiver (http://creativeco mmons.org/publicdomain/zero/1.0/) applies to the data made available in this article, unless otherwise stated in a credit line to the data. 
Conclusions: The presented data highlight the potential value of RBM3 as a predictive biomarker of chemotherapy response in MIBC, which could, if prospectively validated, improve treatment stratification of patients with this aggressive disease.

Keywords: RBM3, Biomarker, Cell cycle, Prediction, Chemotherapy response, Muscle-invasive bladder cancer

\section{Background}

The quest for molecular determinants that could advance our understanding of the biological behaviour of tumour cells, and add prognostic and predictive guidance for refining treatment strategies, has resulted in the characterization of several promising candidates, including RNA-binding motif protein 3 (RBM3).

RBM3, originally discovered as a cold-shock protein [1], has pleiotropic cellular functions. With its DNA and RNA binding capabilities [2], RBM3 promotes global protein synthesis [3], the stability of mRNA bearing AUrich elements [4], and posttranscriptional biogenesis of microRNAs [5], thus exerting broad regulatory influences on the proteome [6]. RBM3 is induced in response to cellular stress, e.g. endoplasmatic reticulum (ER) stress, hypothermia and hypoxia, to mediate cell protection by attenuating both apoptosis and necrosis $[1,7,8]$. This causality has been illuminated within the research context of brain ischemia, where RBM3 has demonstrated an indispensable role in the neuroprotective effects of therapeutic hypothermia after hypoxic ischemia [9]. In addition, RBM3 is described as a proto-oncogene, promoting cell cycle progression and preventing mitotic catastrophe [4]. The RBM3 expression status has been highlighted as a potentially useful biomarker for prognostication and treatment responsiveness in multiple malignancies. High RBM3 expression has been shown to signify an improved prognosis in solid cancers including malignant melanoma [10], colorectal [11, 12], urothelial bladder [13, 14], breast [15], and epithelial ovarian cancer [16] (reviewed in [17]). Contrastingly, in pancreatic cancer, high RBM3 levels correlated to reduced survival [18]. Moreover, in vitro studies have reported decreased sensitivity to chemotherapy after RBM3 suppression in epithelial ovarian and pancreatic cancer cells $[16,18]$.

While upregulation of RBM3 expression in urothelial bladder cancer has been identified as an independent factor of a favourable outcome in studies encompassing tumours of all clinical stages, its prognostic and in particular predictive value in muscle-invasive bladder cancer (MIBC) remains unclear. In MIBC, such biomarkers would be of indisputable importance as the survival benefit of standard treatment with neoadjuvant cisplatin-based chemotherapy (NAC) prior to radical cystectomy is limited to $30-50 \%$ of patients due to chemoresistance [19]. Importantly, NAC treatment has a substantial impact on survival in responding patients, especially in complete responders (i.e. pT0N0), whereas non-responding patients are at risk of severe toxicity and surgical delay [19, 20]. Analysis of the highly heterogeneous genomic landscape of MIBC in the context of chemosensitivity have identified several tumour characteristics that may serve as predictive markers of therapeutic efficacy. Somatic mutations in DNA repair-associated genes, including $A T M, R B 1$ and FANCC [21], and ERBB2 [22] have been associated with response to cisplatinbased chemotherapy. ERCC2 mutations have been shown to be sufficient to drive cisplatin-sensitivity in xenograft models [23] and to correlate with NAC response [24], however not in all studies [22]. Taber et al. recently demonstrated a link between genomic instability driven by chromosomal alterations, indels and $B R C A 2$ mutations and improved response rates, in addition to immune cell infiltration and PD-1 protein expression [25]. Furthermore, molecular subtype-based analyses have yielded contrasting results [26], where basal tumours have been associated with an increased overall survival following NAC treatment [27], while enrichment of non-responders within the basal/squamous subtype has been reported [25]. However, as no robust predictive biomarkers have yet been implemented in clinical use, further profiling of pre-treatment transurethral resection of the bladder (TURB) specimens is needed in order to provide decisive insights into the mechanisms underlying chemotherapy response and identify novel biomarkers that could aid treatment selection [28].

The aim of this study was therefore to examine the putative role of RBM3 as a prognostic and predictive biomarker in relation to NAC in MIBC. To this end, RBM3 protein expression was examined by immunohistochemistry (IHC) in paired primary tumour samples from TURB and cystectomy specimens, respectively, as well as a subset of synchronous lymph node metastases from a consecutive cohort of 145 patients. Furthermore, the potentially modifying effect of RBM3 suppression on chemosensitivity was assessed in vitro, and functional genomics was applied to delineate biological processes associated with RBM3. 


\section{Methods}

\section{Study cohort}

A previously detailed [29] retrospective consecutive series of 145 patients diagnosed with MIBC having undergone TURB and ensuing cystectomy at Skåne University Hospital, Malmö, Sweden, between January 1st 2011 and December 31st 2014, was included in the present study. Paired tissue specimens from TURB $(n=145)$, cystectomy $(n=135)$ and lymph node metastases $(n=27)$ could be retrieved. All cases were histopathologically re-evaluated by a board-certified pathologist (KJ). Clinical information was obtained from medical records. Follow-up started at MIBC diagnosis and ended at death or August 31st 2018. One hundred and fifteen (79.3\%) patients had been diagnosed with de novo MIBC. Prior Bacillus Calmette-Guérin (BCG) treatment was denoted in $13(9.0 \%)$ patients, NAC treatment with methotrexate, vinblastine, adriamycin and cisplatin (MVAC) in 65 (44.8\%) patients and adjuvant chemotherapy in $12(8.3 \%)$ patients. Treatment response was based on pathological evaluation of tissue specimens from radical cystectomy. Complete response (pT0N0) was denoted in $26 / 65(40.0 \%)$ and $6 / 80(7.5 \%)$ patients treated with radical cystectomy with and without prior NAC treatment, respectively. Approval for the study was obtained from the Ethics committee at Lund University (reference number 445-2007), whereby the committee waived the need for informed consent other than the option to opt out. All methods were carried out in accordance with relevant guidelines and regulations.

Tissue microarray construction and immunohistochemistry Tissue microarrays (TMAs) were constructed with triplicate $1 \mathrm{~mm}$ cores from each of the different tissue specimens, i.e. TURB specimens, cystectomy specimens and lymph node metastases, using a semi-automated arraying device (TMArrayer, Pathology Devices, Westminster, MD, USA). All core biopsies were taken from representative tumour areas and when possible from different donor paraffin blocks. Four $\mu \mathrm{m}$ TMA-sections were automatically pretreated with the PT Link system (Dako, Copenhagen, Denmark) with target retrieval solution buffer $\mathrm{pH} 6$, and immunohistochemically stained in an Autostainer Plus (Dako) with the human monoclonal anti-RBM3 antibody (AMAb90655, RRID:AB_2665621, dilution 1:750, Atlas Antibodies AB, Stockholm, Sweden). The specificity of the antibody has been previously validated [16]. RBM3 staining was annotated by two independent observers (SW and KJ) blinded to clinical data. Cases with missing TMA cores or cores with an insufficient amount of tumour cells, in addition to cystectomy specimens from cases with pT0 $(n=35)$, were excluded from the subsequent analyses. RBM3 was predominantly expressed in the tumour cell nuclei, whereby the fraction of nuclear positivity (NF) was categorized as 0 (0-1\%), 1 (2-25\%), 2 (26-50\%), 3(51-75\%) and 4 (>75\%), and the intensity (NI) as 0 (negative), 1 (weak), 2 (moderate) and 3 (strong). In cases with heterogeneous RBM3 intensity, the dominating staining pattern was denoted. A combined nuclear score (NS) was constructed, i.e. a multiplier of NF and NI. As cut off values for dichotomization of RBM3 expression into high versus low could not be established by Classification and regression tree (CRT) analysis, the median value of the NS for each tissue specimen was used for subsequent analyses. IHC images were captured using the VS120 Olympus with OlyVIA software v3.2 (Olympus Corporation, Tokyo, Japan).

\section{Cell culture}

Human bladder cancer cell lines RT4 (grade 1, RRID:CVCL_0036) and T24 (grade 3, RRID:CVCL_0554) were purchased from Sigma-Aldrich (St Louis, MO, USA). The cells were cultured in McCoy's 5a medium supplemented with 10\% fetal bovine serum (FBS), 1\% L-glutamine, $100 \mathrm{U} / \mathrm{mL}$ penicillin and $100 \mu \mathrm{g} / \mathrm{mL}$ streptomycin in a humified $5 \% \mathrm{CO}_{2}$ atmosphere at $37^{\circ} \mathrm{C}$. All reagents for the in vitro experiments were purchased from ThermoFisher Scientific (Waltham, USA) unless stated otherwise.

\section{siRNA transfection}

siRNA transfection was performed in a similar manner as previously described [18]. Bladder cancer cells were seeded in T-25 flasks $\left(5 \times 10^{5}\right.$ cells) and incubated for $24 \mathrm{~h}$ at $37^{\circ} \mathrm{C}$. Next, cells were washed twice in phosphate buffered saline (PBS) and resuspended in growth medium without FBS. Cells were transfected with nontargeting negative siRNA control (Silencer ${ }^{\mathrm{TM}}$ Select Negative control No.1 siRNA, catalog number 4390843) or anti-RBM3 (s11858+s11860) siRNA using Lipofectamine 2000, diluted in OptiMEM to a final siRNA concentration of $25 \mathrm{nM}$. After $4.5 \mathrm{~h}$ the transfection was stopped, the medium changed to full growth medium and the cells were left to recover overnight. The following day, cells were harvested and spun down to pellets. The pellets were either fixated, dehydrated and embedded in paraffin for immunocytochemistry or resuspended in TRIzol and stored at $-20^{\circ} \mathrm{C}$ for qPCR.

\section{Immunocytochemistry}

TMAs were constructed from paraffin-embedded cell pellets of RT4 and T24 cells and immunohistochemically stained according to the same protocol as for the formalin-fixed paraffin-embedded tissue specimens. Representative images were taken using cellSens Dimension software (Olympus) at 20X magnification. 


\section{Quantitative PCR (qPCR)}

The cell samples were thawed and RNA purification was performed using TRIzol with phasemaker tubes according to manufacturer's instructions. RNA cleanup was performed using RNeasy minelute kit (QIAGEN) and the RNA concentration was determined using Qubit with the RNA HS kit. Prior to qRT-PCR, cDNA reverse transcription was performed with the High-capacity cDNA reverse transcription kit and total cDNA concentration was determined using Qubit with the DNA HS kit. Ten ng per reaction of each sample was used for subsequent qRT-PCR with RBM3, CCND1, CCND3, CCNG1, CDK2, CDK4, and CDKN1B TaqMan gene expression assay (Assay ID Hs00943160_g1, Hs00765553_m1, Hs05046059_s1, Hs00171112_m1, Hs01548894_m1, Hs00364847_m1 and Hs00153277_m1, respectively), with samples run in triplicates. $18 \mathrm{~S}$ served as endogenous control (Assay ID Hs039288985_g1).

\section{Cell viability assay}

Following siRNA transfection and $24 \mathrm{~h}$ incubation with regular growth medium, cells were harvested and reseeded in 96 -well plates $\left(2 \times 10^{4}\right.$ cells per well). The following day, cells were subjected to cisplatin $(0-250 \mu \mathrm{M})$ or gemcitabine $(0-250 \mathrm{nM})$ for 24 or $30 \mathrm{~h}$, respectively, in regular growth medium. WST-1 was added to the wells and the plates were read at $450 \mathrm{~nm}$ after $1 \mathrm{~h}$, with a reference wavelength of $620 \mathrm{~nm}$. Cell viability of non-chemotherapy treated siRBM3-transfected and non-targeting siRNA control cells was measured at 24, 30 and $72 \mathrm{~h}$.

\section{Cell cycle analysis}

Cells were plated in 6-well plates $\left(1-2 \times 10^{5}\right)$ and incubated for $72 \mathrm{~h}$ at $37^{\circ} \mathrm{C}$. The cells were transfected with siRNA against RBM3 or non-targeting negative control for $4.5 \mathrm{~h}$. The following day, cells were harvested by trypsinization, counted, washed with PBS and fixated $\left(1 \times 10^{6}\right.$ per sample $)$ in ice cold $70 \%$ ethanol. The samples were stored at $-20^{\circ} \mathrm{C}$ until flow cytometry. Prior to cell cycle analysis, cells were washed with PBS and resuspended in $500 \mu \mathrm{L}$ Propidium Iodide (PI) solution (SigmaAldrich). Samples were run using BD Accuri C6 (BD Biosciences, Mississauga, Canada) and $2 \times 10^{4}$ events were collected of each sample. The cell populations were gated and subjected to doublet discrimination to identify single cells, followed by application of the Watson Pragmatic algorithm for gating of G0/G1, S and G2/M cell populations using FlowJo software v10.6.1.

\section{Western immunoblotting}

Cells were seeded in 6 -well plates $\left(2 \times 10^{5}\right.$ cells per well $)$ and incubated for $48 \mathrm{~h}$ at $37^{\circ} \mathrm{C}$ prior to siRNA-mediated RBM3 silencing. The following day, cells were washed with PBS, lysed on ice for $10 \mathrm{~min}$ in lysis buffer $(10 \mathrm{mM}$ Tris- $\mathrm{HCl}, 50 \mathrm{mM} \mathrm{NaCl}, 5 \mathrm{mM}$ EDTA, $30 \mathrm{mM}$ sodium pyrophosphate, $50 \mathrm{nM}$ sodium fluoride, $100 \mu \mathrm{M}$ sodium orthovanadate, $1 \%$ Triton X100, $\mathrm{pH} 7.6)$ and stored at $-20^{\circ} \mathrm{C}$. Protein quantification was performed using Pierce BCA Protein Assay Kit according to manufacturer's instructions and $20 \mu \mathrm{g}$ was used from each sample. The samples were denatured in Laemmli sample buffer (Sigma-Aldrich), boiled for $5 \mathrm{~min}$ at $95^{\circ} \mathrm{C}$ and placed on an $8-16 \%$ gradient gel (Bio-rad Laboratories, Hercules, USA) with high range rainbow markers at both ends (GE Healthcare Life Sciences). Following electrophoresis, wet tank transfer was performed, and proteins were transferred to a $0.45 \mu \mathrm{m}$ nitrocellulose membrane and dried for $1 \mathrm{~h}$. Total protein staining was performed using Revert 700 (LI-COR Biosciences, Lincoln, USA), imaged at $700 \mathrm{~nm}$. The membrane was destained and blocked with Intercept TBS blocking buffer (LI-COR). Following blocking, the membrane was cut and primary antibody incubation was performed overnight at $4{ }^{\circ} \mathrm{C}$ with antiGAPDH (Millipore 1:1000) or anti-RBM3 (AMAb90655, 1:1000). The membrane was subsequently washed and incubated with secondary IRDye $800 \mathrm{CW}$ goat antimouse (LI-COR) for $1 \mathrm{~h}$ at room temperature (GAPDH 1:15000, RBM3 1:5000). The secondary antibody was thoroughly rinsed off, followed by near-infrared (NIR) protein detection using a LI-COR Biosciences Odyssey Imaging System. Images were analysed using Image studio software (LI-COR). Protein quantification was performed in Empiria Studio Software (LI-COR) by normalizing each lane against total protein content and the relative protein concentration after siRNA transfection compared to control was calculated.

\section{RNA-sequencing}

T24 cells were transfected with anti-RBM3 siRNA or non-targeting siRNA control, as described above. RNA purification was performed according to the $\mathrm{qPCR}$ protocol and samples were prepared in triplicate. RNA quantification and quality assessment were performed using Nanodrop 1000 (Mason Technology, Dublin, Ireland) and Bioanalyzer 2100 (Agilent, Santa Clara, USA). cDNA libraries were prepared from the RNA samples using TruSeq Stranded mRNA Library Prep Kit on the NeoPrep instrument (Illumina, San Diego, USA) according to manufacturer's instructions, and sequenced (single end $1 \times 75 \mathrm{bp}$ ) using the NextSeq 500 platform (Illumina). Fastq files were downloaded from the Illumina BaseSpace using the BaseSpace download tool and the quality of the files was determined using FastQC. Data were trimmed of sequencing adaptors and low-quality base calls using BBDuk tool in the BBMap package. Alignment to the human hg19/GRCh37 genome reference was done 
using STAR version 2.5.2a [30]. Duplicate reads were marked using Picard MarkDuplicates. Read counts were produced by the featureCounts tool from the SubRead package [31], combined for all samples and used as input for analysis of differential gene expression. Differential expression gene (DEG) analysis was conducted using the R package DESeq2 [32]. Gene ontology (GO) enrichment analysis for detection of altered cellular pathways were applied using the Gene Ontology enrichment analysis and visualization tool (GOrilla) [33]. DEGs with fold change \pm 1.5 and false discovery rate (FDR) $<0.01$ were used as input for enrichment analysis. GO terms with Benjamini-Hochberg multiple testing corrected FDR $q$-value $<0.05$ were considered significantly enriched.

\section{Statistical analysis}

Wilcoxon signed-rank test was used for comparison of biomarker expression in paired tissue specimens. Chi-square test and Fisher's Exact test for categorical variables and Mann-Whitney $U$ test for continuous variables were applied to examine associations between RBM3 expression and clinicopathological characteristics. $P$-values were adjusted for multiple testing using the Holm-Bonferroni method. Kaplan-Meier estimates and log-rank tests were used to examine differences in overall survival (OS), cancer-specific survival (CSS) and time to recurrence (TTR) in combined strata according to RBM3 expression and NAC treatment.TTR was defined as time from TURB to the date of recurrent disease or death from bladder cancer. Cox regression proportional hazard models were used to estimate hazard ratios (HRs) for the impact of RBM3 levels on OS, CSS, and TTR in univariable and multivariable analysis, adjusted for age at diagnosis, pathological tumour stage at cystectomy, nodal stage, neoadjuvant, and adjuvant chemotherapy. For assessment of a potential treatment interaction between RBM3 and NAC, an interaction variable was constructed of NAC status $( \pm)$ x dichotomous RBM3 expression (low/high). The interaction term was analysed in relation to OS, CSS and TTR using Cox regression analysis, where the univariable model included NAC status, the binary covariate of RBM3 expression and the interaction variable, and the multivariable model was adjusted for the above-mentioned parameters. For in vitro experiments, unpaired $t$ test and non-linear regression were used. Data are presented as mean \pm SEM derived from at least three independent experiments. Statistical analyses were performed using IBM SPSS Statistics version 25 (SPSS Inc., Chicago, IL, USA) for clinical data, GraphPad Prism version 9 (GraphPad Software, LA Jolla, CA, USA) for experimental data and RStudio Version 1.2.5033 (RStudio Team, Boston, MA, USA) for sequencing data. Graphs were constructed using GraphPad. All statistical tests were two-sided and $p$-values $<0.05$ were considered significant.

\section{Results \\ Longitudinal nuclear RBM3 expression in paired tissue specimens}

Tumour-specific RBM3 protein expression could be evaluated in TURB specimens from 141/145 (97.2\%) cases, in cystectomy specimens from 89/135 (65.9\%) cases and in lymph node metastases from 25/27 (92.6\%) cases. Representative images of RBM3 immunostaining and the distribution of RBM3 expression across tissue samples are shown in Fig. 1a-d. Analysis of RBM3 expression in paired tissue samples was performed using Wilcoxon-signed rank test. For the entire cohort, significantly higher RBM3 expression levels were denoted in TURB specimens compared to cystectomy specimens $(p<0.001)$. There were no significant differences in RBM3 expression between primary tumours and lymph node metastases, neither for TURB nor cystectomy specimens ( $p=0.548$ and $p=0.344$, respectively). After stratification according to NAC treatment, the difference in RBM3 expression between TURB and cystectomy specimens remained significant in NAC-untreated patients $(p<0.001)$, and a similar trend was also indicated in NAC-treated patients $(p=0.053)$ (Additional file 1: Fig. S1a, b).

For subsequent statistical analyses, RBM3 expression was categorized into low versus high expression based on median values of the nuclear score across TMA cores for each case and specimen type (see Fig. 1b). For TURB specimens, the median value was 2.0 , rendering $57 / 141$ (40.4\%) cases with high expression, and for cystectomy specimens the median value was 1.0 , rendering $20 / 89$ $(22.5 \%)$ cases with high expression. Notably, while the RBM3 expression was significantly higher in TURB specimens compared to cystectomy specimens, a shift from low RBM3 expression in TURB specimens to high RBM3 expression in cystectomy specimens was recorded in ten out of $89(11.2 \%)$ cases.

\section{Associations of RBM3 expression with clinicopathological characteristics}

The distribution of patient and tumour characteristics of the study cohort according to RBM3 expression is presented in Additional file 2: Table S1. A sub-analysis of patients from whom paired TURB specimens and cystectomy specimens could be assessed are demonstrated in Additional file 3: Table S2. No significant correlations between biomarker expression and established clinicopathological factors were observed. 


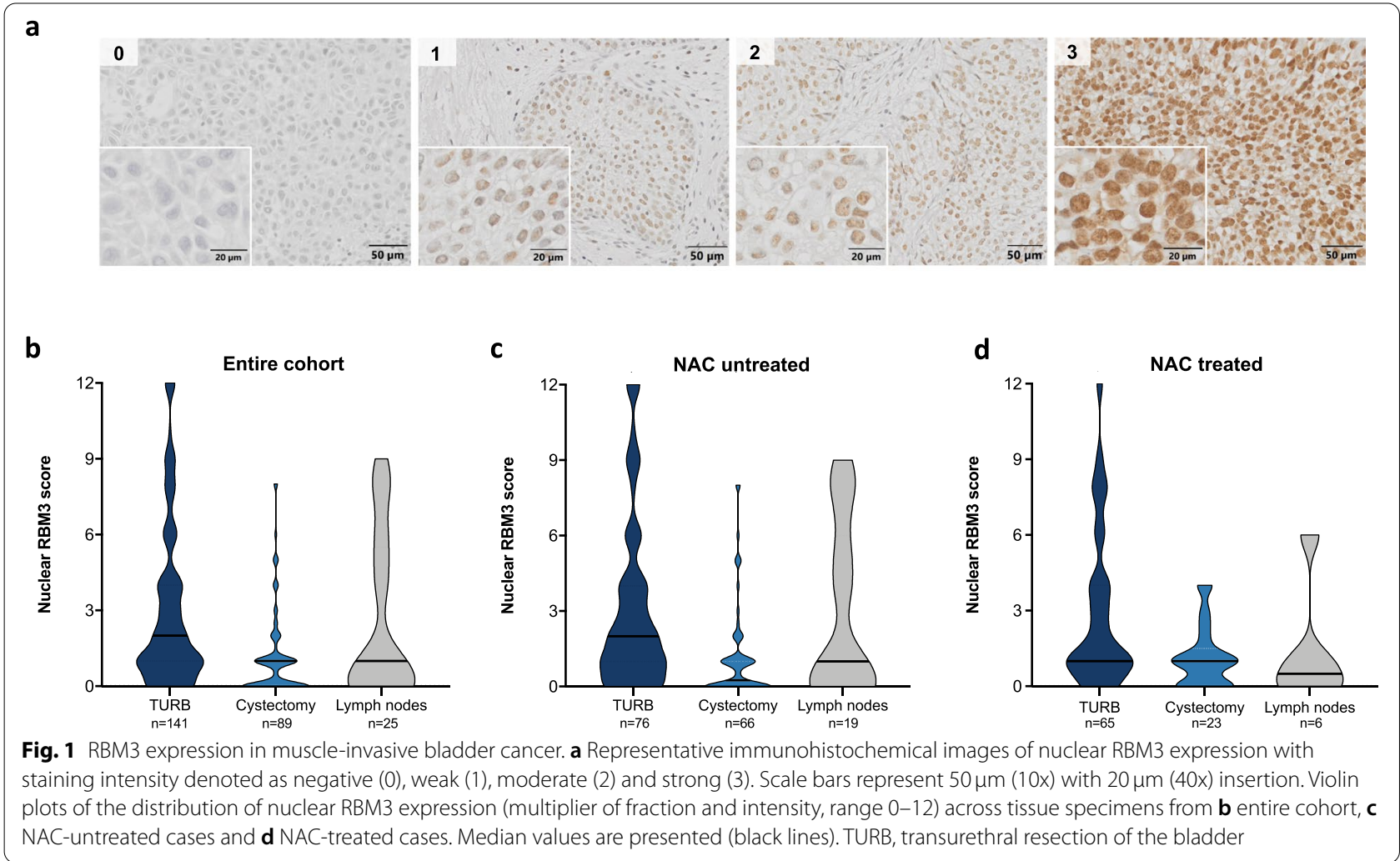

\section{Associations of RBM3 expression with histopathological response}

The correlation between RBM3 expression in TURB specimens and histopathological response to NAC treatment was next evaluated (Fig. 2a). In the entire cohort, downstaging of the primary tumour to $\leq \mathrm{pTa} / \mathrm{CIS}$ was observed in 33/65 (50.8\%) of the NAC-treated patients, out of whom 29/65 (44.6\%) experienced pathological non-invasive downstaging to $\leq \mathrm{pTa}, \mathrm{CIS}$, N0. Further analysis of NAC-treated patients according to RBM3 expression showed that the fraction of pathological downstaging of the primary tumour was higher in patients with high RBM3 expression compared to low RBM3 expression; however, this was not statistically significant $(p=0.156)$. A similar, although less evident, trend was also seen for pathological non-invasive downstaging to $\leq \mathrm{pTa}$, CIS, N0 following NAC.

\section{Prognostic and predictive significance of RBM3 expression}

To assess the potential prognostic and predictive value of RBM3 expression, Kaplan-Meier analyses of OS, CSS and TTR were conducted in combined strata according to biomarker expression in TURB specimens and NAC treatment. At 5-year follow up, 59/141(41.8\%) patients had died, $49 / 59(83.1 \%)$ of whom due to MIBC, and $54 / 141(38.3 \%)$ had denoted recurrent disease. As shown in Fig. 2b, c, NAC-untreated patients with high RBM3 tumoural expression had a significantly reduced OS and CSS compared to NAC-treated patients ( $p=0.001$ and $p=0.002$, respectively). RBM3 expression was not prognostic in relation to OS and CSS in univariable Cox regression analysis (Fig. 2e). In multivariable analysis, adjusted for age at diagnosis, T-stage at cystectomy, $\mathrm{N}$-stage, NAC, and adjuvant chemotherapy that have previously been shown to be prognostic factors for the herein investigated cohort [29], high RBM3 expression was found to be independently associated with an impaired OS $(\mathrm{HR}=1.77$; 95\% CI 1.01-3.13). A similar, however non-significant, trend was observed for CSS $(\mathrm{HR}=1.77$; 95\% CI 0.95-3.29).

Since most local recurrences manifest during the first 24 months and distant metastases within 3years after radical cystectomy [34], analysis of TTR at both 3- and 5-year follow-up was performed. The lowest proportion of recurrence-free patients was observed for NACuntreated patients with high tumoural RBM3 expression, which served as the reference group for pairwise comparison between the investigated strata (Fig. 2d). Interestingly, patients with high tumoural RBM3 expression not receiving NAC had a significantly higher proportion of recurrences compared to NAC-treated patients $(p=0.007)$, where the largest difference in risk of recurrence between 

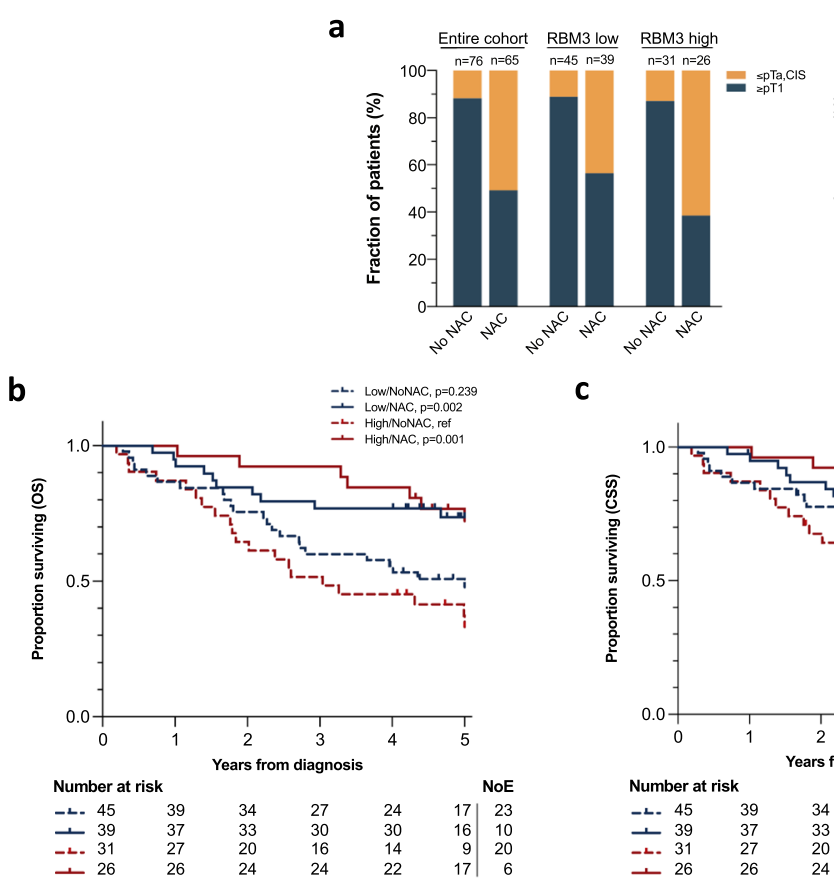

c

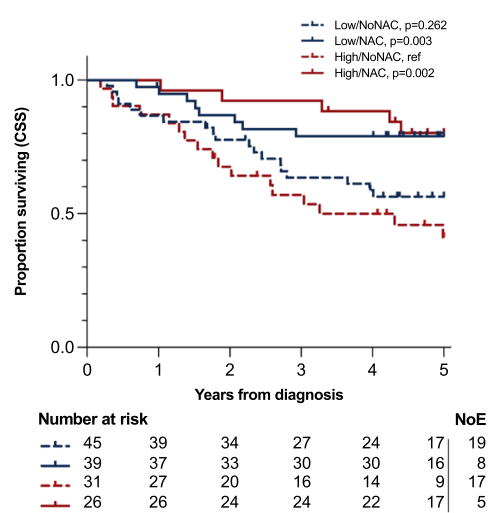

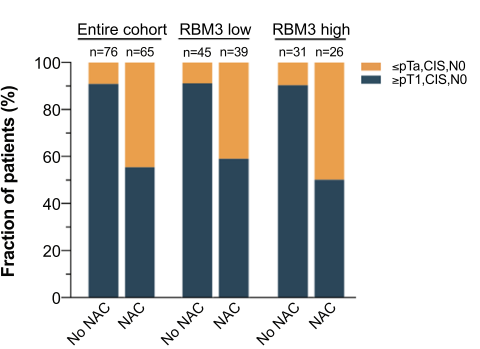

d

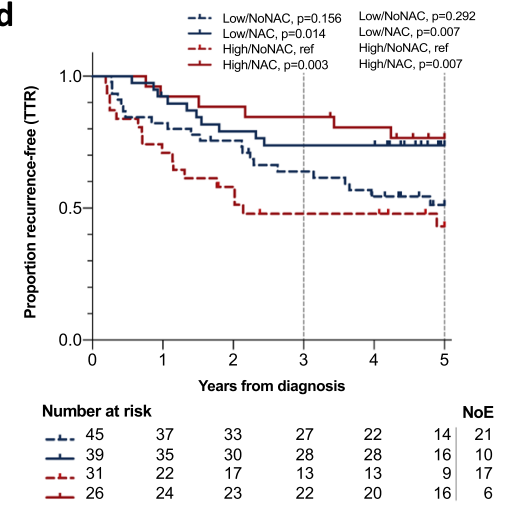

e

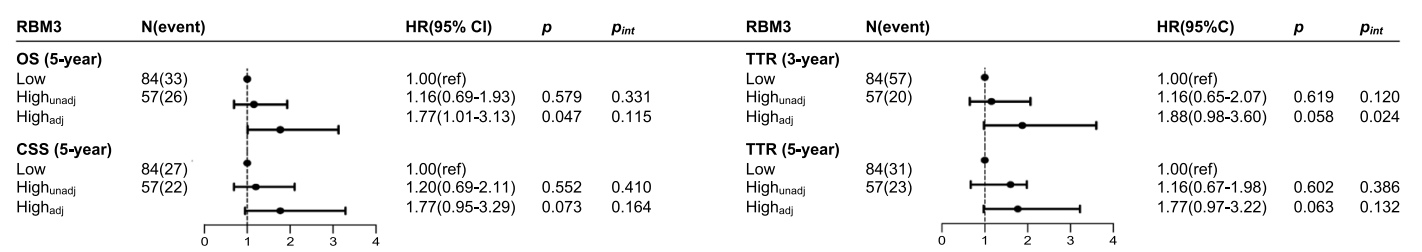

Fig. 2 Histopathological response and risk of recurrence according to neoadjuvant chemotherapy and RBM3 expression. a The proportion of patients with pathological downstaging of the primary tumour (pTO, Ta, CIS) as well as pathological non-invasive downstaging ( $\leq$ pTa, CIS, N0) in the entire cohort and according to RBM3 expression. Kaplan-Meier estimates of b 5-year overall survival (OS), c 5-year cancer-specific survival (CSS) and d 3- and 5-year time to recurrence (TTR) after diagnosis stratified according to dichotomous RBM3 expression in TURB specimens and neoadjuvant chemotherapy. P-values are derived from log-rank test for pairwise comparison, with high RBM3/No NAC as the reference group (ref). NoE, number of events. e Forest plot illustrating hazard ratio with 95\% confidence interval (CI) and $p$-values $(p)$ from uni- and multivariable Cox proportional hazards analysis of 5-year OS, 5-year CSS and 3- and 5-year TTR, respectively. Multivariable model adjusted for age at diagnosis (continuous), T-stage at cystectomy, $\mathrm{N}$-stage, neoadjuvant, and adjuvant chemotherapy. $p_{\text {int }}: p$-value for interaction derived from univariable and multivariable Cox regression analysis of OS, CSS and TTR, respectively, which included a term of interaction by multiplication of NAC status ( \pm ) and the binary covariate of RBM3 expression (low/high)

these patient groups was observed during the first 3 years after diagnosis $(p=0.003)$. In univariable Cox regression analysis of the risk of recurrence of MIBC within 3 and 5 years, respectively, RBM3 expression was not prognostic (Fig. 2e). In multivariable analysis, a trend, however non-significant, towards a higher risk of recurrence was denoted in patients with high $\mathrm{RBM} 3$ expression $(\mathrm{HR}=1.88$; 95\% CI $0.98-3.60$ and $\mathrm{HR}=1.77$; $95 \%$ CI $0.97-3.22$ for 3 and 5 years, respectively). A potential treatment interaction between NAC and RBM3 expression was assessed by inclusion of an interaction term, i.e. multiplier of NAC status (yes/no) and dichotomous RBM3 expression (low/ high), to the univariable and multivariable Cox regression models. No significant treatment interaction between NAC and RBM3 expression could be seen in relation to OS and CSS. However, in relation to TTR, a significant treatment interaction $(p=0.024)$ between NAC and RBM3 expression was observed in the adjusted model during the first 3 years after diagnosis, but did not remain significant in the analysis based on 5-year follow-up (Fig. 2e).

\section{RBM3 suppression impairs sensitivity to chemotherapy in vitro}

Given that RBM3 expression was frequently denoted in the MIBC cohort (84.8\% of patients) and the finding of a reduced risk of recurrence and a prolonged survival in 
NAC-treated patients with high RBM3 expression, we sought to elucidate mechanisms related to RBM3 function in MIBC using an in vitro model of the well-characterized human bladder cancer cell lines RT4 and T24, of which the latter represents invasive disease [35]. At baseline, T24 cells displayed 2.5-fold higher RBM3 mRNA expression levels than RT4 cells ( $p=0.005$, Fig. 3a). The cells were transfected with siRNA targeting RBM3 and non-targeting negative siRNA control. Representative IHC images of siRBM3-transfected and control cells are displayed in Fig. 3b. Following transfection, qRT-PCR and Western blot analyses demonstrated significantly reduced mRNA and protein RBM3 levels (Fig. 3c-d, Original Western blots are presented in Additional file 3: S2).

Next, the potential chemomodulating effect of RBM3 was addressed. Following transfection with siRBM3 and non-targeting negative siRNA control, RT4 and T24 cells were exposed to cisplatin or gemcitabine for 24 and $30 \mathrm{~h}$, respectively, due to the kinetic differences of the two drugs. After $24 \mathrm{~h}$ of incubation with cisplatin, a minor shift towards reduced sensitivity to cisplatin treatment could be observed for RBM3 silenced RT4 cells compared to control. Suppression of RBM3 had no influence on the sensitivity to gemcitabine (Fig. 4a). In T24 cells, RBM3 silencing resulted in a significant 1.5-fold higher half-maximal inhibitory concentration of cisplatin compared to control $\left(\mathrm{IC}_{50} 1.53 \log \mu \mathrm{M}\right.$ and $1.04 \log \mu \mathrm{M}$, respectively, $p<0.001)$. Similarly, when T24 cells were exposed to gemcitabine, siRNA cells displayed a 1.3-fold significant increase in $\mathrm{IC}_{50}$ compared to control (1.70 $\log \mathrm{nM}$ and $1.29 \log \mathrm{nM}$, respectively, $p<0.001$; Fig. 4b). In addition, when non-chemotherapy treated cells were compared to assess the influence on proliferation by silencing of RBM3 alone, no significant differences could be seen between non-targeting siRNA control and siRBM3 transfected RT4 and T24 cells, respectively (Additional file 5: Fig. S3a, b). Therefore, the observed changes in cell viability can be considered to be assigned to altered chemosensitivity after silencing of RBM3.

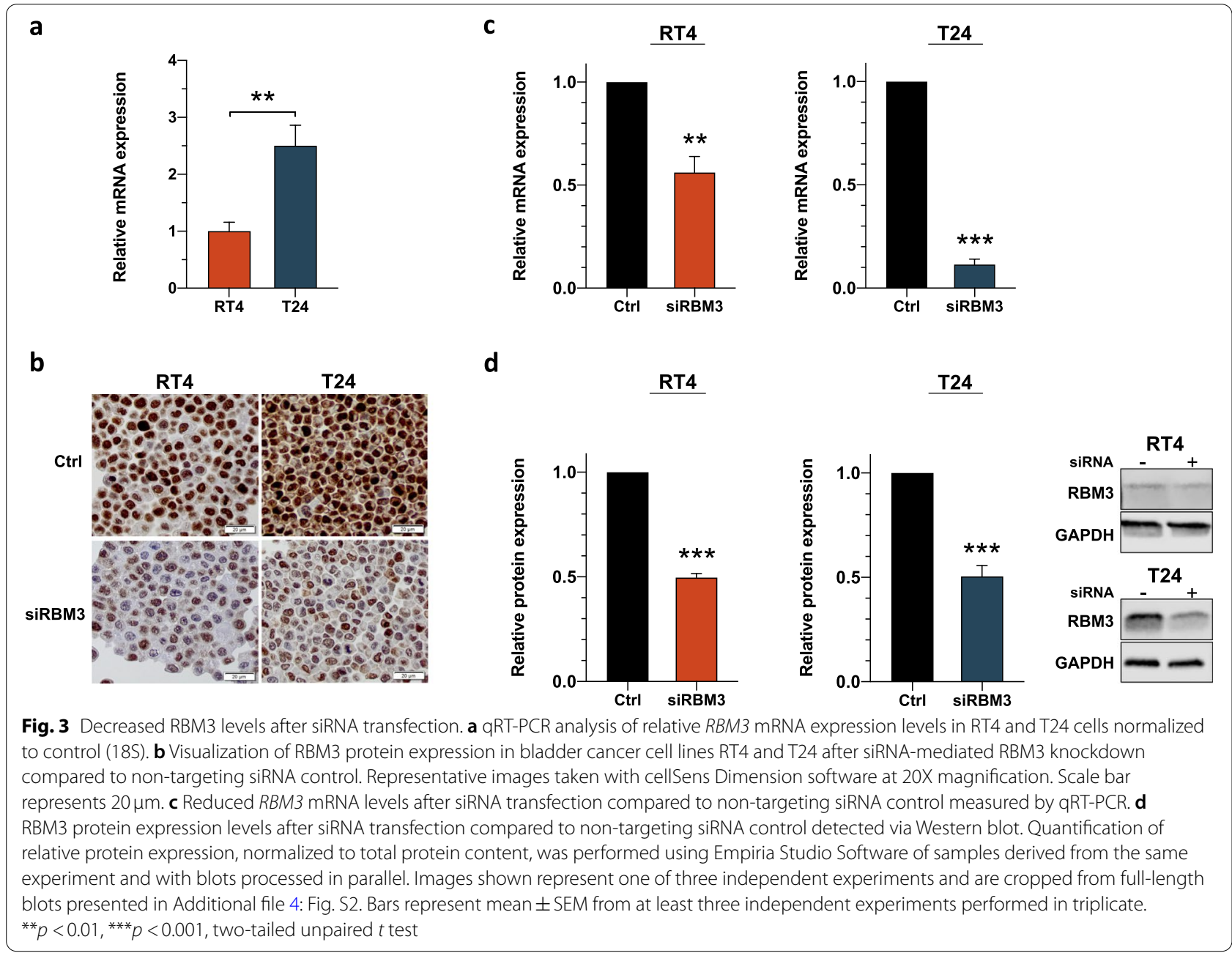




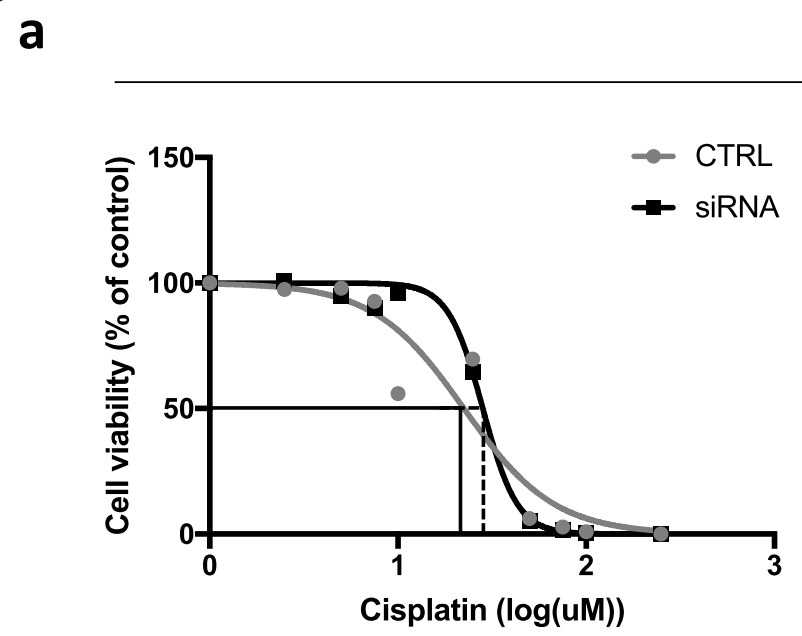

\section{RT4}

b
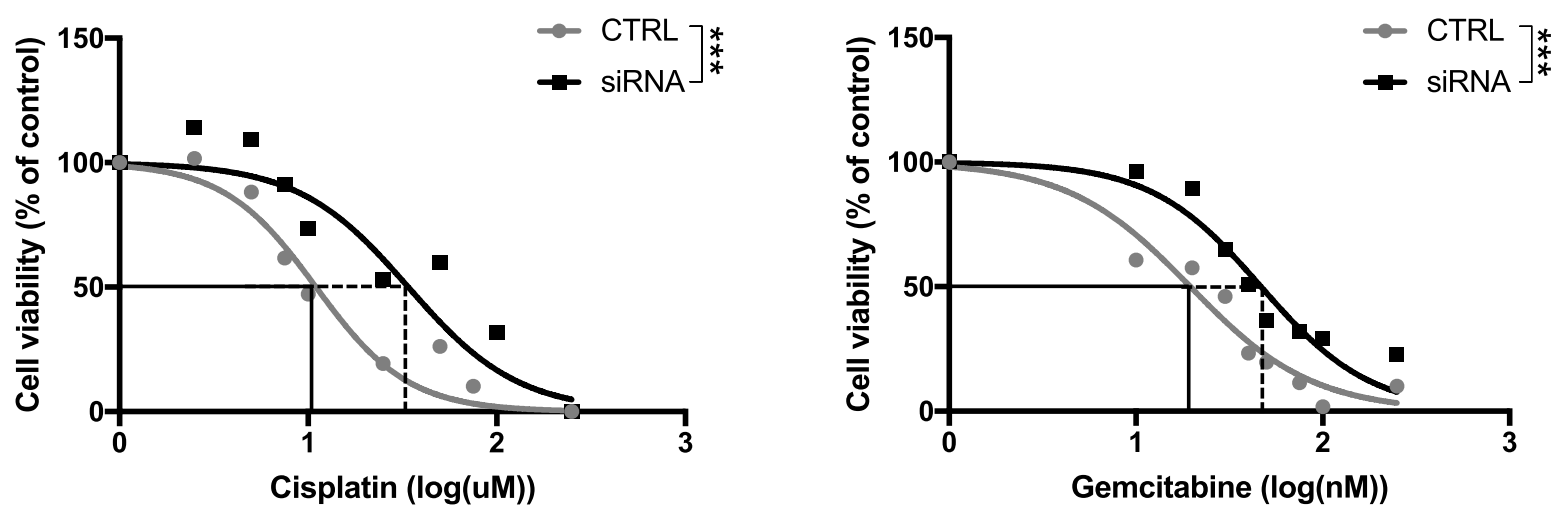

Fig. 4 RBM3 knockdown increases cell viability upon chemotherapy treatment in vitro. Viability of a RT4 and b T24 RBM3 siRNA silenced (black lines) and non-targeting siRNA control (grey lines) treated bladder cancer cells after exposure to increased doses of cisplatin and gemcitabine. Cells

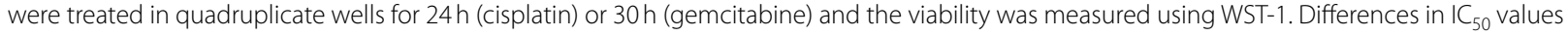
between siRNA and negative control were calculated by non-linear regression of normalized values, relative to control (untreated cells, $100 \%$ cell viability). The graph represents one of three independent experiments. ${ }^{* *} p<0.001$

\section{Mapping of RBM3-associated cellular processes}

In an attempt to decipher the transcriptome level effects of RBM3, the gene expression profiles of siRBM3-treated and control T24 cells were subsequently analysed using RNA-sequencing. This resulted in a large number of DEGs (Fig. 5), where the significantly up- and downregulated DEGs $(n=197$ and $n=145$, respectively) were subjected to further GO analysis using GOrilla. Suppression of RBM3 resulted in significantly enriched GO terms for involvement of downregulated genes in a number of biological processes, including positive regulation of developmental processes, cell cycle processes, positive regulation of the cell cycle, $G_{1} / S$-phase transition, and initiation of DNA replication. The upregulated genes were mainly involved in positive regulation of developmental processes, regulation of cell cycle and cell cycle processes (FDR $<0.05$, Fig. 6a). In cellular component analysis, the significantly DEGs were found to be associated with GO terms for nuclear chromosome part and the minichromosome maintenance (MCM) protein complex (FDR < 0.05, Fig. 6). 


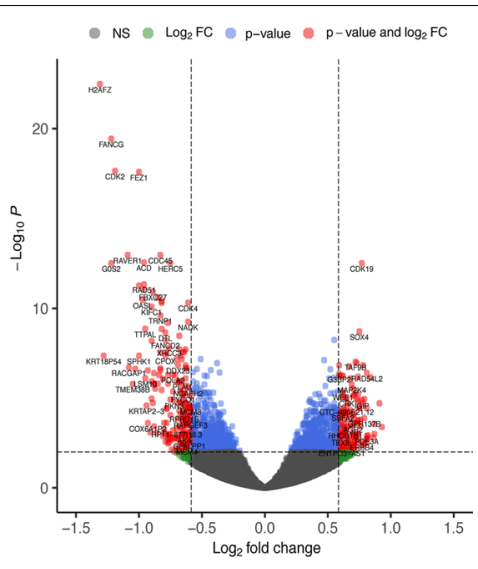

Fig. 5 RBM3 related genes. Volcano plot illustrating differentially expressed genes based on RNA-sequencing of siRBM3-treated and non-targeting siRNA control T24 cells, showing 197 and 145 significantly up- and downregulated genes, respectively, with fold change of $\leq 1.5 / \geq 1.5$, and FDR $<0.01$ (out of total 13,627 genes detected). NS, Not significant. FC, fold change

\section{RBM3 influences $G_{1} / S$-transition}

Following the results from the RNA-sequencing indicating a position of RBM3 in cell cycle regulation, particularly in proceeding from $G_{1}$ to $S$-phase, cell cycle analysis by quantification of DNA content using flow cytometry was applied for further validation (Additional file 6: Fig. S4; Fig. 7a, b). In RT4 cells, RBM3 silencing had no effect on cell cycle distribution. Contrastingly, in T24 cells, a trend towards an accumulation of cells residing in $\mathrm{G}_{1}$-phase $(p=0.119)$ and a corresponding significant decrease in the percentage of cells in S-phase $(p=0.026)$ was demonstrated after siRBM3 transfection. $\mathrm{G}_{1}$-progression is sequentially orchestrated by several cyclins and cyclin-dependent kinases (CDKs), including activation of cyclin D (1, 2 and 3)/CDK4/6 in mid- $G_{1}$, and cyclin $E / C D K 2$ in late $G_{1}$. The catalytic activity of these cyclin/CDK complexes is under regulation of CDK inhibitors of the Ink4 and Cip/Kip family, such as p16, p18, and p27 [36]. The RNA-sequencing analysis of T24 cells indicated a linkage between RBM3 and cell cycle checkpoint markers associated with $G_{1} / S$-phase transition, which was confirmed by qRT-PCR (Fig. 7c). In both cell lines, a significant upregulation of $C C N D 3$ was seen upon siRBM3 treatment compared to control, whereas CDK4 levels were significantly decreased. Additionally, in T24 siRBM3 cells, reduced levels of CCND1 and CDK2 and increased levels of $C D K N 1 B$ were observed compared to control, thus confirming the results from the RNAsequencing. Taken together, these findings suggest a functional role of RBM3 in facilitating cell cycle progression by promoting $\mathrm{G}_{1} / \mathrm{S}$-transition (Fig. $7 \mathrm{~d}$ ).

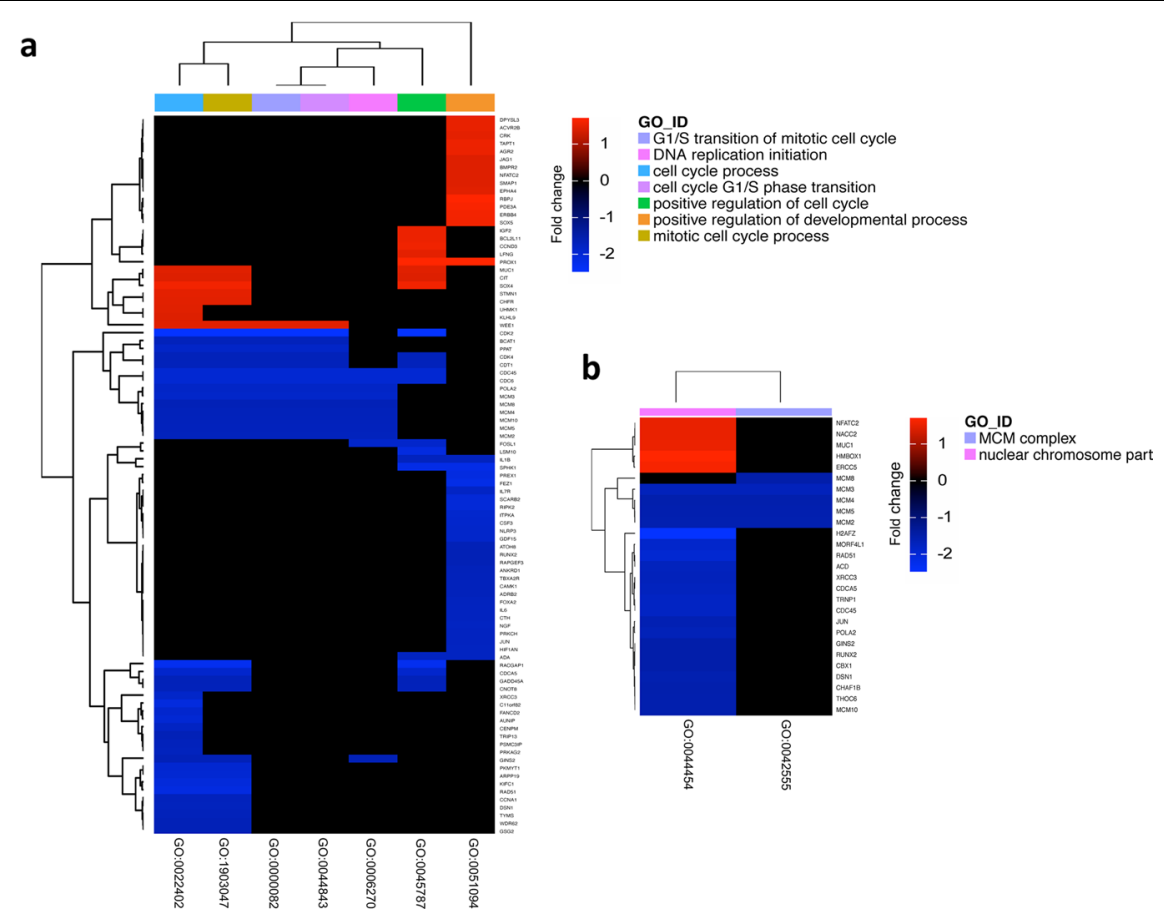

Fig. 6 RBM3 associated biological processes. a Heat map representing biological processes derived from Gene Ontology enrichment analysis (GO terms, $x$-axis) of the significantly up- and downregulated differentially expressed genes ( $y$-axis, FDR $<0.05)$. $\mathbf{b}$ Heat map of the cellular component derived from $\mathrm{GO}$ analysis ( $x$-axis) of the significant differentially expressed genes ( $y$-axis, FDR < 0.05). MCM, mini-chromosome maintenance protein complex 


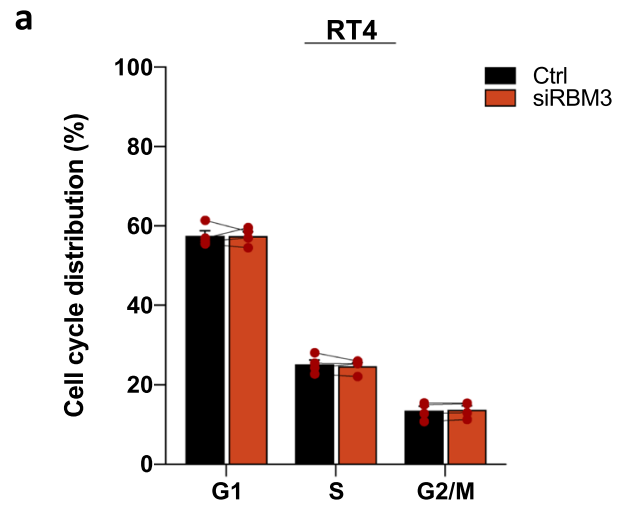

b

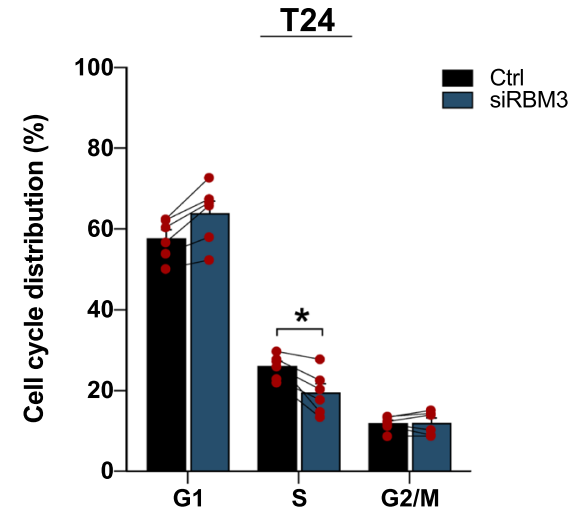

c

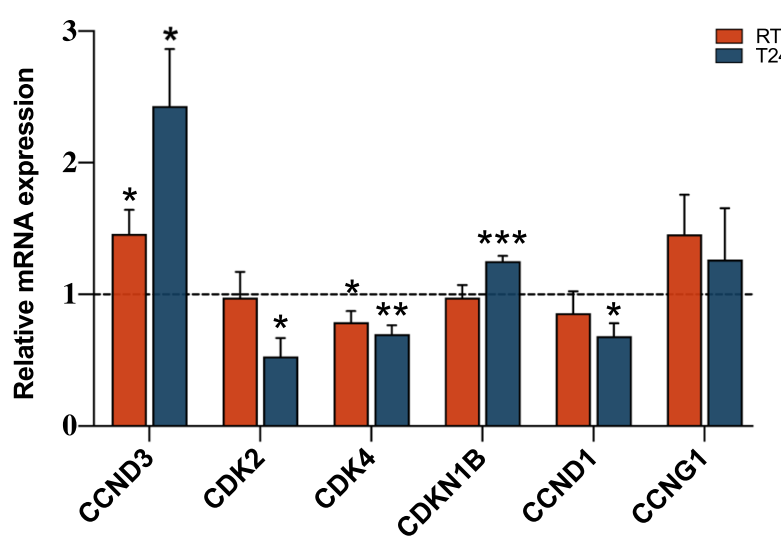

d

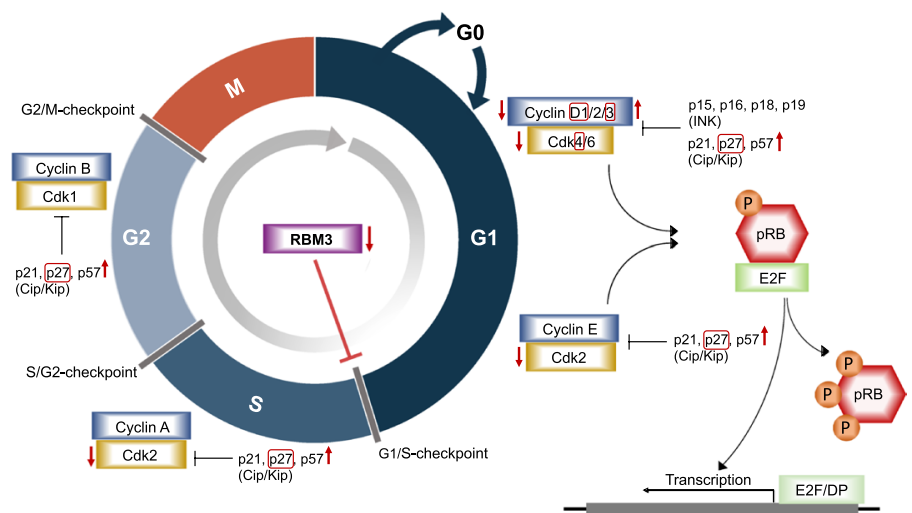

Fig. 7 RBM3 silencing halts cell cycle progression in T24 bladder cancer cells. Following collection of data for $2 \times 10^{4}$ cells, the cell population was gated and doublet discrimination was performed to identify single cells. Cell cycle analysis with the Watson Pragmatic algorithm was applied using FLowJo v10.6.1. on the single cell population for each sample. a In RT4 bladder cancer cells, no effect of RBM3 silencing on cell cycle distribution was observed, as detected by flow cytometry following propidium iodide staining. $\mathbf{b}$ In T24 cells, RBM3 silencing resulted in an increase in $G_{1}$-phase and a significant decrease in S-phase. c Relative mRNA levels of cell cycle checkpoint markers in RT4 and T24 cells transfected with siRNA targeting RBM3 compared to non-targeting siRNA control (dashed line). Mean \pm SEM from at least four independent experiments, ${ }^{*} p<0.05,{ }^{* *} p<0.01$, ${ }^{* * *} p<0.001$, two-tailed unpaired $t$ test. $\mathbf{d}$ A schematic overview of the potential role of RBM 3 in cell cycle progression based on findings from RNA-sequencing, cell cycle analysis as well as measurement of mRNA levels of selected cell cycle markers (red outline) after siRBM3 transfection in T24 cells

\section{Discussion}

In MIBC, interpatient heterogeneity in NAC response constitutes a formidable clinical issue, encouraging the search for accurate predictive biomarkers that could optimize treatment selection and hence, reduce the current overtreatment of non-responders. In the present study, we provide a first description of RBM3 as a potentially predictive biomarker of chemotherapy response in MIBC.

Previous studies have demonstrated upregulation of RBM3 in proliferating non-malignant cells [37] and in malignant compared to normal tissue, with high RBM3 protein expression also having been correlated with favourable prognosis in multiple solid malignancies [17].
Contrastingly, an inverse correlation has been denoted in pancreatic and other periampullary adenocarcinoma, where increased RBM3 protein levels were associated with more aggressive clinicopathological characteristics and a worse prognosis [18], as well as in hepatocellular carcinoma, where high RBM3 levels were shown to promote cellular proliferation, xenografted tumour growth, and signified an impaired survival [38]. In line with these findings, in the herein investigated cohort, patients with high tumour-specific RBM3 expression in TURB specimens had an inferior outcome with an increased risk of having recurrence of MIBC. However, and importantly, patients with high RBM3 expression who received NAC had a significantly reduced risk of recurrence and 
a prolonged survival compared to untreated patients. In addition, a trend towards a higher frequency of pathological downstaging after NAC treatment was observed in patients with high RBM3 expression compared to low expression, posing the question whether RBM3 might enhance chemosensitivity in MIBC. In support of this notion, RBM3 expression status has in several studies been recognized as a predictor of chemoresponsiveness, including gemcitabine and 5-fluorouracil (5-FU) in pancreatic adenocarcinoma [18], oxaliplatin-based chemotherapy in metastatic colorectal cancer [11] as well as of the effect of cisplatin in metastatic testicular non-seminomatous germ cell cancer [39] and epithelial ovarian cancer [16].

The indicated chemomodulating effects of RBM3 were further addressed using an in vitro model of two wellestablished human cancer cell lines commonly used in bladder cancer research. RT4 cells stem from a well-differentiated non-invasive papillary tumour [40], characterized as luminal [41] and p53 wildtype [42], whereas T24 cells are derived from a high-grade invasive transitional cell carcinoma [35], characterized by having mixed basal and luminal molecular features, and p53 mutation [42]. Both cell lines have previously displayed sensitivity to the herein used cytostatic agents [43]. In T24 urothelial cancer cells, which displayed markedly higher RBM3 levels and a more efficient siRNA knockdown on the mRNA level than RT4 cells, siRBM3 transfection resulted in a significantly decreased sensitivity to both cisplatin and gemcitabine. These results are in line with previous studies on pancreatic cancer cells [18], in addition to ovarian cancer, where in vitro studies have shown significantly higher RBM3 levels in A2780 ovarian cancer cells compared to their cisplatin-resistant derivatives, and a decreased sensitivity to cisplatin after siRNA-mediated RBM3 suppression [16]. The cytotoxicity of cisplatin is primarily ascribed its interaction with purine bases of DNA to form crosslinks, resulting in activation of signal transduction pathways involved in DNA damage repair, cell cycle arrest and irreversible apoptotic programs [44]. In epithelial ovarian cancer, a functional description of RBM3 in the maintenance of DNA integrity, including regulation of DNA replication and chromatin remodeling, has been provided, suggesting that the indicated involvement of RBM3 in DNA damage may in part explain the correlation between RBM3 and sensitivity to cisplatin [45]. Moreover, the reduced cytotoxic effect of cisplatin in RBM3 silenced ovarian cancer cells has been reported to be mainly attributed to cell cycle alterations rather than to apoptosis, as cisplatin-induced $\mathrm{G}_{2} / \mathrm{M}$-phase arrest was less evident in siRBM3-treated cells compared to control, whereas no significant changes in the percentage of apoptotic cells could be observed. This hypothesis was further supported by the lacking effect of RBM3 knockdown on the expression levels of the pro-apoptotic protein Bax [16]. Similarly, transcriptomic analysis of T24 cells revealed a functional association between RBM3 expression and cell cycle regulation, particularly at the $\mathrm{G}_{1} / \mathrm{S}$-phase border. This was confirmed by flow cytometry where RBM3 silencing induced $G_{1} / S$-arrest. RBM3 overexpression has previously been shown to relieve cell cycle arrest in the $G_{0} / G_{1}$-phase and cause cell transit into S-phase in neural stem cells [46]. RBM3 knockdown further resulted in altered levels of recognized regulators of $G_{1}$-phase progression and/or $G_{1} / S$-phase transition, including $C D K 2, C D K 4, C C N D 1, C C N D 3$ and $C D K N 1 B$, which gives an insight into potential underlying mechanisms for the observed changes in the cell cycle distribution. In addition, a correlation between RBM3 and initiation of DNA replication was seen, possibly through interaction with the mini-chromosome maintenance (MCM)-complex which serves as a replicative helicase [47]. Thus, while further indepth research is needed, these findings might provide some clues to the mechanistic basis through which RBM3 sensitizes cells to chemotherapy in urothelial carcinoma.

The prognostic implications of RBM3 in urothelial bladder cancer have been evaluated in a few earlier studies of mixed non-muscle-invasive and muscle-invasive tumours. Boman et al. have reported high RBM3 expression to be correlated with clinically less aggressive tumour characteristics and as an independent marker of improved survival $[13,14]$. In a study by Florianova et al. [48], the relationship between RBM3 expression and less advanced tumour stages was confirmed, however, no prognostic significance could be observed. This discrepancy may be due to the use of different IHC assessment strategies, limited follow-up data as well as the larger proportion of advanced tumours in the latter study, as the prognostic value of RBM3 was found to be more evident in non-muscle-invasive tumours [13, 14]. In contrast to the present study, treatment status has not been previously accounted for, thus providing a possible explanation for the paradoxical relationship between upregulation of the proto-oncogene RBM3 and an improved outcome in advanced tumours. The herein presented results accordingly add to the accumulating notion of RBM3 as a predictive biomarker of chemotherapy response, also in MIBC. Yet, additional studies are warranted to elucidate whether high RBM3 expression is associated with a general sensitivity to chemotherapy or is restricted to the herein tested drugs. As for MIBC, the indicated predictive value of RBM3 in relation to gemcitabine merits particular attention. Apart from being recommended in 
combination with cisplatin in the neoadjuvant setting, gemcitabine can be administered to cisplatin-ineligible patients as first-line treatment [49]. Hence, patients with high tumour-specific RBM3 expression who are considered unfit for cisplatin-based chemotherapy may have a benefit from gemcitabine.

Furthermore, analysis of paired tissue samples yielded a higher RBM3 expression in TURB specimens compared to cystectomy specimens. Of note, a shift from low RBM3 levels at time of diagnosis to high RBM3 levels at time of radical cystectomy was shown to be restricted to a limited number of cases, indicating that evaluation of RBM3 expression in TURB specimens is a suitable method in terms of clinical decision-making. Herein, RBM3 expression was analysed using IHC, which offers several advantages over PCR or sequencing in the clinical setting as it is an easier, faster and less costly method. Moreover, IHC allows for biomarker analysis in a subcellular context, which is particularly relevant as it appears to be mainly the nuclear expression of RBM3 that carries prognostic significance in bladder cancer $[13,14]$. RBM3 mRNA levels were not assessed, however, comparison of RBM3 mRNA and protein levels has previously shown concordance [16, 18]. Further studies are needed to investigate if this also applies for RBM3 expression in MIBC.

The results from the present study stem from a consecutive well-annotated cohort of patients. In regard to the distribution of pathological/clinical T-stages at diagnosis and the administered chemotherapy, i.e. all NAC-treated patients having received MVAC regimen, it represents a homogenous study cohort which may explain why the response rates to NAC treatment are in the higher interval of previously reported data $[20,25,50]$. In terms of limitations, these are in line with the known limitations of a retrospective and experimental design. We acknowledge the potential influence of other factors on the prognostic benefit observed in NAC-treated patients with high tumoural RBM3 expression compared to untreated patients. As NAC-untreated patients were older [29] and considered ineligible for chemotherapy, they could have a worse prognosis regardless. As performance status was rarely registered in the medical records, no definitive conclusions can be made, and this should thus be denoted in subsequent studies. However, the included patients were considered fit for extensive surgical treatment and the prognostic analyses were based on TTR and CSS in addition to OS, limiting the influence of comorbidity on mortality. Future larger studies are needed to validate the results from this study, preferably also in relation to previously reported gene signature profiles and molecular subtypes of MIBC that have been shown to carry predictive significance [21-25]. In addition, follow-up studies of the mechanistic effect of RBM3 in MIBC could provide further valuable insights into its suggested role in chemosensitivity, e.g. by using additional cell lines representative of muscle-invasive disease and exploration of downstream pathways next to cell cycle progression.

\section{Conclusions}

The herein presented clinical data provide a clear indication of the beneficial effect of neoadjuvant chemotherapy in urothelial tumours expressing high nuclear RBM3 levels. An observation further corroborated by in vitro experiments where siRBM3 transfected T24 high-grade urothelial cancer cells displayed a reduced sensitivity to both cisplatin and gemcitabine.

Transcriptomic analysis revealed a functional description of RBM3 in facilitating cell cycle progression by promoting $G_{1} / S$-phase transition in these cells. Future investigations are of significant interest in order to further characterize the mechanisms of action of RBM3 as well as to delineate the potential clinical utility of RBM3 as a predictive biomarker of chemotherapy response in MIBC.

\section{Abbreviations \\ Cl: Confidence interval; DEGs: Differentially expressed genes; GO: Gene ontol- ogy; HR: Hazard ratio; IHC: Immunohistochemistry; MCM: Mini-chromosome maintenance protein complex; MIBC: Muscle-invasive bladder cancer; NAC: Neoadjuvant chemotherapy; RBM3: RNA-binding motif protein 3; TMA: Tissue microarray; TTR: Time to recurrence; TURB: Transurethral resection of the bladder.}

\section{Supplementary Information}

The online version contains supplementary material available at https://doi. org/10.1186/s12885-021-09168-7.

Additional file 1: Figure S1. RBM3 protein expression in paired tissue specimens of MIBC. Spaghetti plots of the distribution of nuclear RBM3 expression in paired tissue specimens in a) the entire cohort and b) stratified according to NAC treatment. P-values were calculated using Wilcoxon signed-rank test, significant $p$-values are highlighted in bold. TURB, transurethral resection of the bladder.

Additional file 2: Table S1. Associations between RBM3 expression and clinicopathological characteristics in the entire cohort.

Additional file 3: Table S2. Sub-analysis of the associations between RBM3 expression and clinicopathological characteristics in strata according to TURB-only and paired tissue specimens.

Additional file 4: Figure S2. Original Western blots used for Fig. 3d. Three sets of samples from independent experiments were run in parallel separated by ladders for a) RT4 and b) T24 cells, respectively. Left images show total protein content with Revert total protein stain. Red line indicates cut prior to antibody incubation and detection. Right images display protein expression for loading control and protein of interest after detection. The red boxes indicate the cropped regions used in Fig. $3 \mathrm{~d}$.

Additional file 5: Figure S3. Comparison of cell viability after siRBM3 transfection. Cell viability of siRBM3 transfected a) RT4 and b) T24 bladder cancer cells compared to control (non-targeting siRNA) at 24, 30 and $72 \mathrm{~h}$ 
after transfection measured by WST-1 assay. No significant differences in cell viability were observed after siRBM3 transfection. Data represent mean \pm SEM from at least three independent experiments performed in triplicate.

Additional file 6: Figure S4. Cell cycle analysis of RT4 and T24 bladder cancer cells. a) Representative flow cytometry scatter plots visualizing the gating strategy for cell population identification and doublet discrimination. Following transfection of RT4 and T24 cells with siRBM3 or nontargeting control, data were collected for $2 \times 10^{4}$ cells for each sample, the cell population was gated and doublet discrimination was performed to identify single cells. b) The Watson Pragmatic algorithm was applied for identification of $\mathrm{G} 1, \mathrm{~S}$ and $\mathrm{G} 2 / \mathrm{M}$ cell populations.

\section{Acknowledgements}

Not applicable.

\section{Authors' contributions}

SW: Methodology, formal analysis, writing-original draft, review and editing. KB: Conceptualization, methodology, writing-original draft, review and editing. BM: Methodology, writing-review and editing. BN: Methodology, writing-review and editing. W.M.G: Resources, writing-review and editing. EK: Conceptualization, methodology, formal analysis, writing-review and editing. KJ: Conceptualization, supervision, formal analysis, funding acquisition, writing-original draft, review and editing. All authors read and approved the final manuscript.

\section{Funding}

Open access funding provided by Lund University. This study was supported by grants from the Swedish Research Council (grant no. 201503598/2018-02441), the Kamprad Family Foundation, the Governmental Funding of Clinical Research within the National Health Service (ALF, no. F2014/354), Lund University Faculty of Medicine, the Irish Cancer Society Collaborative Cancer Research Centre BREAST-PREDICT (CCRC13GAL), the Science Foundation Ireland (SFI) under the Investigator Programme OPTiPREDICT (15/IA/3104), and the Strategic Research Programme Precision Oncology Ireland (18/SPP/3522). The funding sources had no involvement in study design, collection, analysis or interpretation of data, or in the writing of the report.

\section{Availability of data and materials}

RNA-sequencing data are deposited at the NCBI Gene Expression Omnibus database [https://www.ncbi.nlm.nih.gov/geo/] under the accession number GSE167558. Access to all other data generated or analysed during this current study will be evaluated according to Swedish legislation and be made available from the corresponding author on reasonable request.

\section{Declarations}

\section{Ethics approval and consent to participate}

The study was approved by the Ethics committee at Lund University (reference number 445-2007). In accordance with national legislation and institutional requirements, the committee waived the need for informed consent other than the option to opt out. All methods were carried out in accordance with relevant guidelines and regulations.

\section{Consent for publication}

Not applicable.

\section{Competing interests}

W.M. Gallagher is a co-founder, shareholder and Chief Scientific Officer in OncoMark Limited, and reports receiving commercial research grants from and is an unpaid Scientific Advisory Board member with Carrick Therapeutics. The other authors declare no competing interest.

\section{Author details}

'Division of Oncology and Therapeutic Pathology, Department of Clinical Sciences, Lund University, Lund, Sweden. ${ }^{2}$ UCD School of Biomolecular and Biomedical Sciences, UCD Conway Institute, University College Dublin, Belfield, Dublin, Ireland.

Received: 16 May 2021 Accepted: 24 December 2021

Published online: 02 February 2022

\section{References}

1. Danno S, Nishiyama H, Higashitsuji H, Yokoi H, Xue JH, Itoh K, et al. Increased transcript level of RBM3, a member of the glycine-rich RNA-binding protein family, in human cells in response to cold stress. Biochem Biophys Res Commun. 1997;236(3):804-7.

2. Wright $C F$, Oswald BW, Dellis $S$. Vaccinia virus late transcription is activated in vitro by cellular heterogeneous nuclear ribonucleoproteins. J Biol Chem. 2001;276(44):40680-6.

3. Smart F, Aschrafi A, Atkins A, Owens GC, Pilotte J, Cunningham BA, et al. Two isoforms of the cold-inducible mRNA-binding protein RBM3 localize to dendrites and promote translation. J Neurochem. 2007;101(5):1367-79.

4. Sureban SM, Ramalingam S, Natarajan G, May R, Subramaniam $\mathrm{D}$, Bishnupuri KS, et al. Translation regulatory factor RBM3 is a proto-oncogene that prevents mitotic catastrophe. Oncogene. 2008;27(33):4544-56

5. Pilotte J, Dupont-Versteegden EE, Vanderklish PW. Widespread regulation of miRNA biogenesis at the dicer step by the cold-inducible RNA-binding protein, RBM3. PLoS One. 2011;6(12):e28446.

6. Pilotte J, Kiosses W, Chan SW, Makarenkova HP, Dupont-Versteegden E, Vanderklish PW. Morphoregulatory functions of the RNA-binding motif protein 3 in cell spreading, polarity and migration. Sci Rep. 2018;8(1):7367.

7. Wellmann S, Bührer C, Moderegger E, Zelmer A, Kirschner R, Koehne P, et al. Oxygen-regulated expression of the RNA-binding proteins RBM3 and CIRP by a HIF-1-independent mechanism. J Cell Sci. 2004;117(Pt 9):1785-94.

8. Zhu X, Zelmer A, Kapfhammer JP, Wellmann S. Cold-inducible RBM3 inhibits PERK phosphorylation through cooperation with NF90 to protect cells from endoplasmic reticulum stress. FASEB J. 2016;30(2):624-34.

9. Zhu X, Yan J, Bregere C, Zelmer A, Goerne T, Kapfhammer JP, et al. RBM3 promotes neurogenesis in a niche-dependent manner via IMP2-IGF2 signaling pathway after hypoxic-ischemic brain injury. Nat Commun. 2019;10(1):3983.

10. Jonsson L, Bergman J, Nodin B, Manjer J, Pontén F, Uhlén M, et al. Low RBM3 protein expression correlates with tumour progression and poor prognosis in malignant melanoma: an analysis of 215 cases from the Malmö diet and cancer study. J Transl Med. 2011;9:114.

11. Siesing C, Sorbye H, Dragomir A, Pfeiffer P, Qvortrup C, Pontén F, et al. High RBM3 expression is associated with an improved survival and oxaliplatin response in patients with metastatic colorectal cancer. PLoS One. 2017;12(8):e0182512.

12. Hjelm B, Brennan DJ, Zendehrokh N, Eberhard J, Nodin B, Gaber A, et al. High nuclear RBM3 expression is associated with an improved prognosis in colorectal cancer. Proteomics Clin Appl. 2011;5(11-12):624-35.

13. Boman K, Andersson G, Wennersten C, Nodin B, Ahlgren G, Jirström K. Podocalyxin-like and RNA-binding motif protein 3 are prognostic biomarkers in urothelial bladder cancer: a validatory study. Biomark Res 2017:5:10.

14. Boman K, Segersten U, Ahlgren G, Eberhard J, Uhlén M, Jirström K, et al. Decreased expression of RNA-binding motif protein 3 correlates with tumour progression and poor prognosis in urothelial bladder cancer. BMC Urol. 2013;13:17.

15. Jögi A, Brennan DJ, Rydén L, Magnusson K, Fernö M, Stål O, et al. Nuclear expression of the RNA-binding protein RBM3 is associated with an improved clinical outcome in breast cancer. Mod Pathol. 2009;22(12):1564-74.

16. Ehlén A, Brennan DJ, Nodin B, O'Connor DP, Eberhard J, Alvarado-Kristensson $\mathrm{M}$, et al. Expression of the RNA-binding protein RBM3 is associated with a favourable prognosis and cisplatin sensitivity in epithelial ovarian cancer. J Transl Med. 2010;8:78.

17. Zhou RB, Lu XL, Zhang CY, Yin DC. RNA binding motif protein 3: a potential biomarker in cancer and therapeutic target in neuroprotection. Oncotarget. 2017;8(13):22235-50. 
18. Karnevi E, Dror LB, Mardinoglu A, Elebro J, Heby M, Olofsson SE, et al. Translational study reveals a two-faced role of RBM3 in pancreatic cancer and suggests its potential value as a biomarker for improved patient stratification. Oncotarget. 2018;9(5):6188-200.

19. Lee FC, Harris W, Cheng HH, Shenoi J, Zhao S, Wang J, et al. Pathologic response rates of gemcitabine/cisplatin versus methotrexate/vinblastine/ adriamycin/cisplatin neoadjuvant chemotherapy for muscle invasive urothelial bladder cancer. Adv Urol. 2013;2013:317190.

20. Rosenblatt R, Sherif A, Rintala E, Wahlqvist R, Ullén A, Nilsson S, et al. Pathologic downstaging is a surrogate marker for efficacy and increased survival following neoadjuvant chemotherapy and radical cystectomy for muscle-invasive urothelial bladder cancer. Eur Urol. 2012;61(6):1229-38.

21. Plimack ER, Dunbrack RL, Brennan TA, Andrake MD, Zhou Y, Serebriiskii IG, et al. Defects in DNA repair genes Predict response to neoadjuvant cisplatin-based chemotherapy in muscle-invasive bladder cancer. Eur Urol. 2015;68(6):959-67.

22. Groenendijk FH, de Jong J, Fransen van de Putte EE, Michaut M, Schlicker $A$, Peters D, et al. ERBB2 mutations characterize a subgroup of muscleinvasive bladder cancers with excellent response to neoadjuvant chemotherapy. Eur Urol. 2016;69(3):384-8.

23. Li Q, Damish AW, Frazier Z, Liu D, Reznichenko E, Kamburov A, et al. ERCC2 helicase domain mutations confer nucleotide excision repair deficiency and drive cisplatin sensitivity in muscle-invasive bladder cancer. Clin Cancer Res. 2019;25(3):977-88

24. Van Allen EM, Mouw KW, Kim P, lyer G, Wagle N, Al-Ahmadie H, et al. Somatic ERCC2 mutations correlate with cisplatin sensitivity in muscleinvasive urothelial carcinoma. Cancer Discov. 2014;4(10):1140-53.

25. Taber A, Christensen E, Lamy P, Nordentoft I, Prip F, Lindskrog SV, et al. Molecular correlates of cisplatin-based chemotherapy response in muscle invasive bladder cancer by integrated multi-omics analysis. Nat Commun. 2020;11(1):4858.

26. Kamoun A, de Reyniès A, Allory Y, Sjödahl G, Robertson AG, Seiler R, et al. A consensus molecular classification of muscle-invasive bladder cancer. Eur Urol. 2020;77(4):420-33.

27. Seiler R, Ashab HAD, Erho N, van Rhijn BWG, Winters B, Douglas J, et al. Impact of molecular subtypes in muscle-invasive bladder cancer on predicting response and survival after neoadjuvant chemotherapy. Eur Urol. 2017;72(4):544-54.

28. Takata R, Katagiri T, Kanehira M, Tsunoda T, Shuin T, Miki T, et al. Predicting response to methotrexate, vinblastine, doxorubicin, and cisplatin neoadjuvant chemotherapy for bladder cancers through genome-wide gene expression profiling. Clin Cancer Res. 2005;11(7):2625-36.

29. Wahlin S, Nodin B, Leandersson K, Boman K, Jirström K. Clinical impact of T cells, B cells and the PD-1/PD-L1 pathway in muscle invasive bladder cancer: a comparative study of transurethral resection and cystectomy specimens. Oncoimmunology. 2019;8(11):e1644108.

30. Dobin A, Davis CA, Schlesinger F, Drenkow J, Zaleski C, Jha S, et al. STAR: ultrafast universal RNA-seq aligner. Bioinformatics. 2013;29(1):15-21.

31. Liao Y, Smyth GK, Shi W. featureCounts: an efficient general purpose program for assigning sequence reads to genomic features. Bioinformatics. 2014;30(7):923-30.

32. Love MI, Huber W, Anders S. Moderated estimation of fold change and dispersion for RNA-seq data with DESeq2. Genome Biol. 2014;15(12):550.

33. Eden E, Navon R, Steinfeld I, Lipson D, Yakhini Z. GOrilla: a tool for discovery and visualization of enriched $\mathrm{GO}$ terms in ranked gene lists. BMC Bioinformatics. 2009;10:48.

34. Huguet J. Follow-up after radical cystectomy based on patterns of tumour recurrence and its risk factors. Actas Urol Esp. 2013;37(6):376-82.

35. Bubeník J, Baresová M, Viklický V, Jakoubková J, Sainerová H, Donner J. Established cell line of urinary bladder carcinoma (T24) containing tumour-specific antigen. Int J Cancer. 1973;11(3):765-73.

36. Donjerkovic D, Scott DW. Regulation of the $\mathrm{G} 1$ phase of the mammalian cell cycle. Cell Res. 2000;10(1):1-16.

37. Wellmann S, Truss M, Bruder E, Tornillo L, Zelmer A, Seeger K, et al. The RNA-binding protein RBM3 is required for cell proliferation and protects against serum deprivation-induced cell death. Pediatr Res. 2010;67(1):35-41.

38. Dong W, Dai ZH, Liu FC, Guo XG, Ge CM, Ding J, et al. The RNA-binding protein RBM3 promotes cell proliferation in hepatocellular carcinoma by regulating circular RNA SCD-circRNA 2 production. EBioMedicine. 2019;45:155-67.
39. Olofsson SE, Nodin B, Gaber A, Eberhard J, Uhlén M, Jirström K, et al. Low RBM3 protein expression correlates with clinical stage, prognostic classification and increased risk of treatment failure in testicular nonseminomatous germ cell cancer. PLoS One. 2015;10(3):e0121300.

40. Crallan RA, Georgopoulos NT, Southgate J. Experimental models of human bladder carcinogenesis. Carcinogenesis. 2006;27(3):374-81.

41. Warrick JI, Walter V, Yamashita H, Chung E, Shuman L, Amponsa VO, et al. FOXA1, GATA3 and PPARy cooperate to drive luminal subtype in bladder cancer: a molecular analysis of established human cell lines. Sci Rep. 2016;6:38531.

42. Zuiverloon TCM, de Jong FC, Costello JC, Theodorescu D. Systematic review: characteristics and preclinical uses of bladder cancer cell lines. Bladder Cancer. 2018;4(2):169-83.

43. da Silva GN, de Castro Marcondes JP, de Camargo EA, da Silva Passos Júnior GA, Sakamoto-Hojo ET, Salvadori DM. Cell cycle arrest and apoptosis in TP53 subtypes of bladder carcinoma cell lines treated with cisplatin and gemcitabine. Exp Biol Med (Maywood). 2010;235(7):814-24.

44. Siddik ZH. Cisplatin: mode of cytotoxic action and molecular basis of resistance. Oncogene. 2003;22(47):7265-79.

45. Ehlén $\AA$, Nodin B, Rexhepaj E, Brändstedt J, Uhlén M, Alvarado-Kristensson $M$, et al. RBM3-regulated genes promote DNA integrity and affect clinical outcome in epithelial ovarian cancer. Transl Oncol. 2011;4(4):212-21.

46. Yan J, Goerne T, Zelmer A, Guzman R, Kapfhammer JP, Wellmann S, et al. The RNA-binding protein RBM3 promotes neural stem cell (NSC) proliferation under hypoxia. Front Cell Dev Biol. 2019;7:288.

47. Bochman ML, Schwacha A. The mcm complex: unwinding the mechanism of a replicative helicase. Microbiol Mol Biol Rev. 2009;73(4):652-83.

48. Florianova L, Xu B, Traboulsi S, Elmansi H, Tanguay S, Aprikian A, et al. Evaluation of RNA-binding motif protein 3 expression in urothelial carcinoma of the bladder: an immunohistochemical study. World J Surg Oncol. 2015;13:317.

49. Alfred Witjes J, Lebret T, Compérat EM, Cowan NC, De Santis M, Bruins HM, et al. Updated 2016 EAU guidelines on muscle-invasive and metastatic bladder cancer. Eur Urol. 2017;71(3):462-75.

50. Grossman HB, Natale RB, Tangen CM, Speights VO, Vogelzang NJ, Trump $\mathrm{DL}$, et al. Neoadjuvant chemotherapy plus cystectomy compared with cystectomy alone for locally advanced bladder cancer. N Engl J Med. 2003;349(9):859-66.

\section{Publisher's Note}

Springer Nature remains neutral with regard to jurisdictional claims in published maps and institutional affiliations.
Ready to submit your research? Choose BMC and benefit from:

- fast, convenient online submission

- thorough peer review by experienced researchers in your field

- rapid publication on acceptance

- support for research data, including large and complex data types

- gold Open Access which fosters wider collaboration and increased citations

- maximum visibility for your research: over $100 \mathrm{M}$ website views per year

At BMC, research is always in progress.

Learn more biomedcentral.com/submissions 